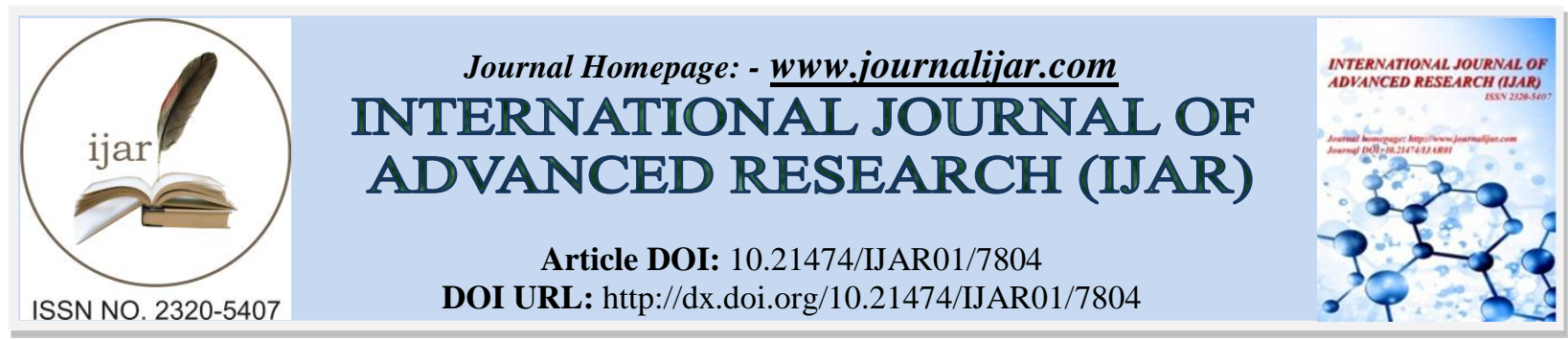

RESEARCH ARTICLE

\title{
A STUDY ON WEARABLE DEVICES FOR THE SAFETY AND SECURITY OF A GIRL CHILD AND WOMEN.
}

\section{P. Nandhini and K. Moorthi.}

1. PG Scholar, Department of Computer Science and Engineering, Jansons Institute of Technology, Coimbatore, Tamil Nadu, India.

2. Assistant Professor, Department of Computer Science and Engineering, Jansons Institute of Technology, Coimbatore, Tamil Nadu, India.

\section{Manuscript Info}

(..........................

Manuscript History

Received: 4 August 2018

Final Accepted: 6 September 2018

Published: October 2018

Keywords:

Child, Women, Safety, Security,

Trafficking, Harassment, IOT.

\begin{abstract}
Child and Women play vital roles in our society from their birth to the end of life. In the past few years, crime against child and women has increased to a great extent. They are facing many securities related problems. We can hear the news of women harassments than their achievements. Trafficking in women and children is one of the worst abuses of human rights. But it is very difficult to estimate the scale of the phenomenon as trafficking is closely related to child labour, bonded labour, child marriage, kidnapping and abduction and prostitution even though these phenomena can exist also independent of trafficking. In such situations, they are helpless and don't have any way to protect them or inform it to their family members, neighbours or police station and they feel as handicaps. In order to overcome such problems several apps and smart security systems like armband, bracelet, jacket, smart toy, smart watch and wristband are being implemented for both child and women. This survey paper covers various techniques and systems proposed by the various researchers and analyse the merits and demerits of those systems. By overcoming the drawbacks of the existing systems, we proposed a new system based on IOT.
\end{abstract}

Copy Right, IJAR, 2018. All rights reserved.

\section{Introduction}

The misbehaviours against children and women are increasing day by day at an exponential rate. They are under the threat of easily being kidnapped. In today's date, women and a child face physical harassment in public places, schools and at workplaces or while traveling. Rape is the fourth most common crime against Women in India. According to latest National Crime Records Bureau (NCRB) 2017 annual report, 56,709 rape cases are reported across only India. The number of reported rape cases has been steadily increasing over the past decade. Most cases of physical harassment take place when women are alone or while traveling. Women feel insecure to step outside their house. There are many android applications for smartphones but for those who don't use smartphones or those who cannot keep their mobile handy at their workplace, hence many other systems are developed. 
The Internet of Things System (1OT) refers to the set of devices and systems that stay interconnected with real-world sensors and actuators to the Internet. The motivation for this wearable comes from the increasing need for safety for little children in current times as there could be scenarios of the child getting lost in the major crowded areas. These papers focusses on the key aspect that lost child can be helped by the people around the child and can play a significant role in the child's safety until reunited with the parents. Most of the wearables available today are focused on providing the location, activity, etc. of the child to the parents via Wi-Fi and Bluetooth. But Wi- Fi and Bluetooth seem a very unreliable source to transfer information. Therefore, it is intended to use SMS as the mode of communication between the parent and child's wearable device, as this has fewer chances of failing compared to WiFi and Bluetooth.

The platform on which these projects will be running on is the Arduino Uno or Arduino Nano microcontroller board based on the ATmega328P, and the functions of sending and receiving SMS, calls and connecting to the internet which is provided by the Arduino GSM shield using the GSM network. Also, additional modules employed which will provide the current location of the child to the parents via SMS. We can make use of number of sensors to precisely detect the real time situation of the women in critical abusive situations.

\section{Literature Survey}

Seelam and Prasanti(2018)[1] says that in today's world women are less secure and have many issues regarding their security purpose. This paper describes about safe and secured electronic system for women which comprises of an Arduino controller and sensors such as temperature LM35, flex sensor, MEMS accelerometer, pulse rate sensor, sound sensor. A buzzer, LCD, GSM and GPS are used in this project. When the woman is in threat, the device senses the body parameters like heartbeat rate, change in temperature, the movement of victim by flex sensor, MEMS accelerometer and the voice of the victim is sensed by sound sensor. When the sensor crosses the threshold limit the device gets activated and traces the location of the victim using the GPS module. By using the GSM module, the victim's location is sent to the registered contact number. The connection is shown below in fig. 1 .

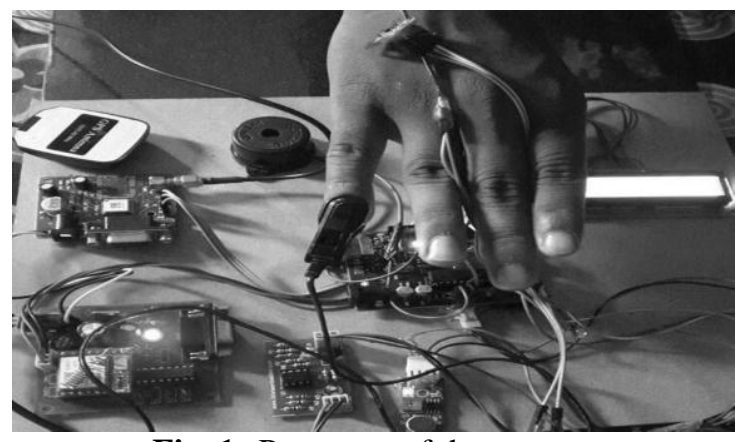

Fig. 1:-Prototype of the system.

Prof. Kiran et al(2017)[2] says that, in today's world, security is the major issue for an individual. In this project the system consists of a monitoring device, which gets activated when the device is tapped upon then a text message along with voice alert message is received by the respective emergency contacts. Further the person who receives the notifications can find and track the location without the interaction of the victim's application at each and every function.

Harikiran et al(2016)[3] propose to have a device which is the integration of multiple devices, hardware comprises of a wearable "Smart band" which continuously communicates with Smart phone that has access to the internet. The application is programmed and loaded with all the required data which includes Human behaviour and reactions to different situations like anger, fear and anxiety. This generates a signal which is transmitted to the smart phone. The software or application has access to GPS and Messaging services which is pre-programmed in such a way that whenever it receives emergency signal, it can send help request along with the location co-ordinates to the nearest Police station, relatives and the people in the near radius who have application. This action enables help instantaneously from the Police as well as Public in the near radius who can reach the victim with great accuracy.

Sai Prashanth et al (2017)[4] developed an application that incorporates all the unique features such as real-time location tracking and integrate all the features offered by the existing system such as GPS tracking, SOS. The 
application requires an initial registration along with emergency contacts and the user is asked to update the emergency contacts from time to time. When the user is travelling from one place to another, the dynamic GPS tracking offered by PubNub's channel is turned on to view the user's location on a map. Users with the same app can monitor other users with this app through the dynamic GPS Tracking system through the PubNub channel. When the SOS button is pressed then an alert message which contains the name of the user, GPS Location and a help message is sent via SMS. The user has access to first-aid information and toll free helpline phone numbers. All the information and data is integrated with Firebase.

Sathyapriya and Mary(2018)[5] describes safety electronic device for women, a wearable smart bracelet, that sends alerts to friends, family, as well as the police when they fell they are in problem. The smart device based on IOT uses a low-energy Bluetooth connection to synchronize to an application on the wearer's Smartphone. The application lets the wearer inform her situation in case of a critical situation - to her friends, family members, the police, or a group. The software or application has access to GPS/GSM and Messaging services, which is preprogrammed in such a way that whenever it receives emergency signal, it can send help request along with the location coordinates to the nearest Police station, relatives using emergency keys (SOS). This action enables help instantaneously from the Police who is in the near geographical location, who can reach the victim with great accuracy. The app also uses the Smartphone's record the incident and subsequently transmits the wearer's location along with the audio recording to the police.

Pawar et al(2018)[6] made an attempt to solve the problems of women safety. The scope of their system is to develop a smart device which can help women in some emergency situations. The system is a smart wearable device which resembles a jacket. The device contains different modules such as GPS (Global Positioning System), GSM (Global System for Mobile communication), Camera, Buzzer, Shock Mechanism Circuit. The main objective of the system is to provide a reliable security system for a woman when they are alone or feel unsafe.

Katerina Demetzou et al(2017)[7] designed a "Smart Bear" which is a hypothetical connected-smart toy for children. While the functionalities it presents are appealing to both children and their parents, the privacy concerns that are raised should be taken into serious consideration. A big amount of personal data of the child (and probably of other uninformed minors and adults in physical vicinity) are processed and analysed, an accurate profile of the child is created and direct marketing practices would most probably take place. The toy could suddenly turn into a surveillance device, while malicious third parties might hack the device and proceed to activities that would even threaten the child's physical and/or mental health.

Helen et al(2017)[8], designed a wearable smart watch. When a women or child wearing this 'watch me' is exposed to sexual or vulnerable attack, the sensor present in it detects the heart beat rate of a person which will be high at the moment by the secretion of epinephrine hormone from hpa axis and gets activated, this will not only provide alarm sound to the attention of nearby people, it will automatically make a call to our registered contact and also through GPS/GSM it will detect the nearby police station and make a ring there so it will be helpful for police to arrive soon at the spot by tracking the GPS, such a system will lead to safer and better environment.

Bhanupriya and Sundarajan(2017)[9] proposed a device which is integrated with multiple devices, comprising of wearable "Activity Tracker Wrist Band" which is programmed with all the required data which includes the behaviour of the human reactions like anger, anxiety, nervousness and fear. When these situations are faced by the victim, the various sensors generate the emergency signals which are to be transmitted to the smart phone. The system effectively monitors the children presence within the expected zone. When the person crosses the monitoring zone, then based on IOT Monitoring system, GSM sends help request by sending messages to the nearest police station, parents and the people in the near radius.

Bhavale et al(2016)[10] proposing a system that works on the controversy of children and women security using IOT. The proposed system intends to a device wireless technique in the form of embedded device namely Arduino for women that will serve the purpose of alerts and way of communicating with secure channels and it captures the image using electronic camera. There are many android applications for women safety but they as not as much as efficient. So to solve this issue of women safety they develop a wireless sensor kit which is easy to use and which is efficient to provide help to that victim. so when the victim press kits button, our application will capture the photo, collect user's information to send notification to registered phone numbers with link of captured image. This saves the time and that victim get help without loss of time. Also in the case of Children security the system proposes a 
speed monitoring and location tracking facilities using GPS, GPRS, GSM. The system consists of bus unit. The bus unit which is used to detect the path of Bus by using GPS. Weather the bus is travelling on its day to day route and also it monitors the over speeding of bus. For the mechanism of vehicle tracking Haversine and Trilateration algorithm are used. According to that by using GSM, alert messages will be send to their parents and vehicle owner. The system has been developed on web based data driven application that provides the useful information.

Anand Jatti et al (2016)[11] presents a work. The aim of this work is to develop a wearable device for the safety and protection of women and girls. This objective is achieved by the analysis of physiological signals in conjunction with body position. The physiological signals that are analysed are galvanic skin resistance and body temperature. Body position is determined by acquiring raw accelerometer data from a triple axis accelerometer. Acquisition of raw data is then followed by activity recognition which is a process of employing a specialized machine learning algorithm. Real-time monitoring of data is achieved by wirelessly sending sensor data to an open source Cloud Platform. Analysis of the data is done on MATLAB simultaneously. This device is programmed to continuously monitor the subject's parameters and take action when any dangerous situation presents itself. It does so by detecting the change in the monitored signals, following which appropriate action is taken by means of sending notifications/alerts to designated individuals. A prototype of the model is shown below in fig. 2.

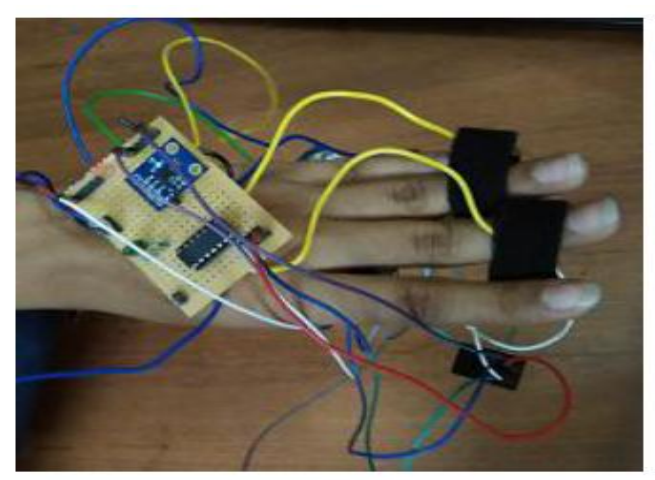

Fig. 2:-prototype model.

Moodbidri and Shahnasser(2017)[12] discusses the concept of a smart wearable device for little children. The major advantage of this wearable over other wearable is that it can be used in any cell phone and doesn't necessarily require an expensive smartphone and not a very tech savvy individual to operate. The purpose of this device is to help parents locate their children with ease. At the moment there are many wearables in the market which help track the daily activity of children and also help find the child using Wi-Fi and Bluetooth services present on the device. But Wi-Fi and Bluetooth appear to be an unreliable medium of communication between the parent and child. Therefore, the focus of this paper is to have an SMS text enabled communication medium between the child's wearable and the parent as the environment for GSM mobile communication is almost present everywhere.

Velayutham et al (2016)[13] developed a system that helps the women and child to seek help in any critical situation. For that, the system contains GPS to detect location and GSM mechanisms to pass their current location to any one of the trusted contacts as a google map link and services are provided to track the locations from that moment onwards to save the person.

Toney et al(2015)[14] says that, with increasing atrocities on women and children, arises the need of an advanced system to serve the purpose of alerting someone for help. Most of the cases remain mystery because of lack of evidences or them being tweaked. The situation is noxious and they propose a system that would aid the victims not only to send a panic and alert message but also collect evidences in the form of images. They propose a system initiated by a human action. It is also given with an option of switch button and a fall detector to activate the system. The armband would have a controller with GSM/GPS kit interfaced. The band would also be interfaced with a wireless camera for collecting images. A human action would initiate the system. On initiation, the video collected is live video streamed to the Control room. An alert message along with the location is sent to a predefined Mobile Station until the system is reset. Since change in Longitude and Latitude is sent continuously; the person can be tracked. The system is designed also to be used as an alert system during medical emergency. 
Niti shree(2016)[15] proposes an Android based solution to aid parents to track their children in real time. Different devices are connected with a single device through channels of internet. The concerned device is connected to server via internet. The device can be used by parents to track their children in real time or for women safety. The proposed solution takes the advantage of the location services provided by GSM. It allows the parents to get their child's location on real time by SMS. Here, a prototype model (device) is created which is simulation based. The work comprises ARM-7 LPC2148 as microcontroller, along with GPS and GSM module. Embedded C core compile using Keil and virtual simulation check using Proteus 8.1 is done. A server is created which will collect all the data generated by our prototype system and send the same to server using GPRS. A Dummy server will be created by using Filezilla. This device will also have the facility of Emergency help key (SOS), if anyone presses the key, automatic help message will be sent to 3 registered mobile numbers on Server.

Jyothi et al (2018)[16] proposes an SMS and E-mail based solution to aid parents to track their children in real-time. Different devices are connected with a single device through channels of internet-concerned parents to track their children in real time or for women safety can use the device. It allows the parents to get their child's location on real time by an E-mail and SMS. Here prototype model (device) is created which is simulation based. The work comprises Raspberry Pi as a microprocessor along with GPS, E-mail and SMS gateway. Python 2.7 Idle compile is used for the purpose of compilation. A server is created which will collect all the data generated by our prototype system and send the same to server using GPRS. A Raspberry Pi camera is being used to capture the surrounding area's images when the child is in trouble.

Pawade and Gaikwad(2015)[17] proposed, a children tracking system based on android terminals. Recently, all over the world crime against the children in the age of 14 to 17 years is more popular. Parent's always worry about their children whenever they are outside from the home. In this paper, the proposed system consists of two sides out of them one is parent module and another is the child module. The child module consists of ARM7microcontroller (LPC 2148), GPS (Global Positioning System), GSM (Global System for Mobile Communication) and voice chip where the parent module consists of android mobile phone. This paper gives the information about missing child from school campus. There are two android mobile phones for the safety of the both module. The system tracking the child from source to destination i.e. from home to school or anywhere.

Pramod et al(2018)[18] designed a security device. The main objective of this work is to create a wearable IOT device for the security and shielding of women, girl children. This is accomplished by the examination of physiological signs in concurrence with body gestures. The signs are analysed and body temperature is measured by galvanic skin resistance. This work deals with body temperature and stress and skin resistance and relationship between them. By applying the records, activities and persons position is analysed. The device makes an analysis of skin resistance and body temperature to analyse the situation of the person.

Pavithra, and Sangeetha(2018)[19] says that the main contribution of the paper is to develop a wearable arm band for safety and protection of women and girls. This objective is achieved by the analysis of physiological signal in conjunction with body position. The physiological signals that are analysed are pulse rate sensor, vibration sensor and if there is any fault it additionally uses a fault detection sensor. Acquisition of raw data makes the Arduino controller function by activating the GPS to send alert messages via GSM and the wireless camera captures images and videos and sends images to the pre-decided contacts and also shares video calling to the family contact. The alarm is employed to alert the surroundings by its sound and meanwhile, she can also use a TAZER as a self-defence mechanism.

Gopperundevi et al(2018)[20] says that the women and child safety is a very important issue due to rising crimes against women these days. This paper proposes to track the women or child from dangerous Situation. The flex sensor band wears by the child or a woman. If they are in dangerous situation they can bend the flex sensor literally, then it will send the accurate location to the android phone by the use of GPS and GSM. The GPS and GSM module which is already connected to the ARDUINO UNO. It converts the analog signal from the flex sensor to the message as a format of google link to our contacts.

\section{Proposed Model}

This work describes about safe and secured electronic system for women which comprises of an Arduino controller and sensors such as temperature LM35, flex sensor, pulse rate sensor. A buzzer, LCD, GSM and GPS are used in this project. The device is mainly built to save a child from harassments. We are placing the touch sensor in the bad 
touching places of the girl child. If any touches on the touch sensor is detected, the device senses the body parameters like heartbeat rate, change in temperature, the movement of victim by flex sensor. When the sensor crosses the threshold limit the device gets activated and traces the location of the victim using the GPS module. By using the GSM module, the victim's location is sent to the registered contact number. A hidden camera is also fixed along with the child dress, when the device gets activated, the camera starts working and it transmits the live scenario to the registered contacts, so that they can be able to see what's happening there. The architecture of our system is shown in the below fig. 3 .

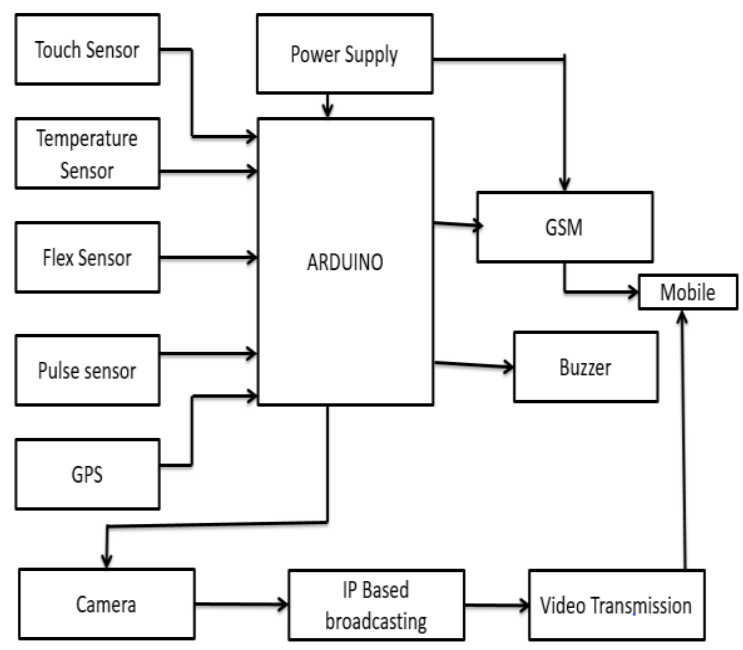

Fig. 3:-Architecture of the Proposed System

\section{Conclusion}

This survey is all about the existing applications of the system for child and women security and comes out with an innovative idea for security and protection. More research is possible with introducing smart technology where people and objects form a network. This will help to solve them technologically with compact equipment and ideas. In these system, an embedded hardware has been developed to collect video streams from the camera front end. The video streams are further processed to activate the whole system. The system also establishes an internet connection that enables the system to connect with smartphone. Using screaming alarms and also alerting the emergency contacts by sending the messages with the location is helpful for child and women's security. This sort of systems can overcome the fear that scares every woman in the country about her safety and security.

\section{Future Enhancement}

Following are a few different paths that can be followed to improve the system developed in this project.

1. Incorporate the advancements made in the field of wearable electronics to develop a more compact device that could possibly be integrated into clothing.

2. Tackle the concern that arises because of the requirement of internet at all points.

3. Incorporating the ability to work in any environment. 


\section{References}

1. Kalpana seelam, k. Prasanti," a novel approach to provide protection for women by using smart security device", ieee international conference on inventive systems and control (icisc 2018), isbn:978-1-5386-0806-7, 2018.

2. Prof. Kiran. Mensinkai, chaitra b.v, chinmayi v pandith, goutam p nayak and jyothsna. C. S, "an intelligent safety system for individual's security", ieee international conference on energy, communication, data analytics and soft computing, 2017.

3. G c harikiran, karthik menasinkai, suhas shirol, "smart security solution for women based on internet of things (iot)", ieee international conference on electrical, electronics, and optimization techniques (iceeot), volume: 3 , 2016.

4. Dantu sai prashanth, gautam patel, dr. B. Bharathi, "research and development of a mobile-based women safety application with real-time database and data-stream network", ieee international conference on circuits power and computing technologies, 2017.

5. T. Sathyapriya, r. Auxilia anitha mary, "women's safety measures through sensor device using iot", international journal of advance research, ideas and innovations in technology volume 4, issue 2, issn: 2454132x, 2018.

6. Rachana b. Pawar, manali h. Kulabkar, kirti s. Pawar, akshata r. Tambe, prof. Smita khairnar, "smart shield for women safety", international research journal of engineering and technology (irjet), volume: 05 issue: 04 , apr2018.

7. Katerina demetzou, leon böck, obaida hanteer, "smart bears don't talk to strangers: analysing privacy concerns and technical solutions in smart toys for children", ieee, 2017.

8. A. Helen, m. Fathima fathila, r. Rijwana, kalaiselvi. V. K. G, "a smart watch for women security based on iot concept "watch me"”, 978-1-5090-6221-8/17/\$31.00_c 2017, ieee.

9. T. Bhanupriya, dr. T. Vp. Sundarajan, "activity tracker wrist band for children monitoring using iot", international journal on recent and innovation trends in computing and communication, issn: 2321-8169, volume: 5 , issue: 11 , november 2017.

10. Ms. Deepali m. Bhavale, ms. Priyanka s. Bhawale, ms. Tejal sasane, mr. Atul s. Bhawale, "iot based unified approach for women and children security using wireless and gps", international journal of advanced research in computer engineering \& technology (ijarcet), issn: 2278 - 1323, volume 5, issue 8, august 2016.

11. Anand jatti, madhvi kannan, alisha rm, vijaya lakshmi p, shrestha sinha, "design and development of an iot based wearable device for the safety and security of women and girl children", ieee international conference on recent trends in electronics information communication technology, may 20-21, 2016.

12. Akash moodbidri, hamid shahnasser, "child safety wearable device", ieee, lcoin, 2017.

13. Dr. Velayutham. R, sabari. M, sorna rajeswari. M, “an innovative approach for women and children's security based location tracking system", international conference on circuit, power and computing technologies [iccpct], ieee, 2016.

14. Glenson toney, dr. Fathima jabeen, puneeth s, "design and implementation of safety armband for women and children using arm7", ieee, 2015.

15. Niti shree, "a review on iot based smart gps device for child and women safety applications", international journal of engineering research and general science, issn 2091-2730, volume 4, issue 3, may-june, 2016.

16. A jyothi, alapati srimaithri, anusha $\mathrm{p}$, avula sindura $\mathrm{s}$, santhosh kumar s, "development of wearable device for the safety and security of women and children", international journal of scientific research in computer science, engineering and information technology, volume 4, issue 6, issn: 2456-3307, 2018.

17. Rita h. Pawade, dr. Arun n. Gaikwad, "android based children tracking system", international journal of science, engineering and technology research (ijsetr), volume 4, issue 6, june 2015.

18. M. Pramod, ch v. Uday bhaskar and k. Shikha, "iot wearable device for the safety and security of women and girl child", international journal of mechanical engineering and technology (ijmet), scopus indexed, volume 9, issue 1, pp. 83-88, january 2018.

19. R. Pavithra, p. S. Sangeetha, "design and implementation of a rescue system for the safety of women by using arduino controller", international journal of advance research, ideas and innovations in technology, issn: 2454132x, volume 4, issue 2, 2018.

20. T. Gopperundevi, r. Manimozhi and r. Nivetha, "high accuracy sensor based women and child safety by using gsm", international journal of advanced scientific research \& development, vol. 05, spl. Iss. 01, ver. Iii, pp. 264 -273 , mar 2018, issn: 2395-6089. 